INTERSTITIAL LUNG DISEASE

\title{
Is endosonography guided fine needle aspiration (EUS- FNA) for sarcoidosis as good as we think?
}

\author{
S M Wildi, M A Judson, M Fraig, W E Fickling, N Schmulewitz, S Varadarajulu, S S Roberts, \\ P Prasad, R H Hawes, M B Wallace, B J Hoffman
}

Thorax 2004;59:794-799. doi: 10.1136/thx.2003.009472

See end of article for authors' affiliations

Correspondence to: Dr B J Hoffman, Professor of Medicine, Digestive Disease Center, Medical University of South Carolina, Charleston, SC 29425, USA; hoffmanb@ musc.edu

Received 12 May 2003 Accepted 3 May 2004
Background: Preliminary data show that endosonography guided fine needle aspiration (EUS-FNA) may be an accurate method for diagnosing sarcoidosis. However, these data were obtained in a small selected group of patients with a very high pretest probability of sarcoidosis. This retrospective study reports on the use of EUS-FNA in an unselected group of patients with mediastinal lymphadenopathy of unknown origin. Methods: The EUS database of a single tertiary referral centre was reviewed for patients who underwent EUS-FNA for mediastinal lymphadenopathy of unknown origin. Clinical presentation and imaging studies of each case were carefully reviewed and the diagnosis "sarcoidosis" or "no sarcoidosis" attributed if possible. The diagnoses were compared with the result of EUS-FNA.

Results: One hundred and twenty four patients were investigated. In 35 cases EUS-FNA identified granulomas (group 1); in the other 89 cases (group 2) no granulomas were detected. The definite diagnoses in group 1 were sarcoidosis $(n=25)$, indefinite $(n=7)$, no sarcoidosis $(n=3)$. The definite diagnoses in group 2 were sarcoidosis $(n=3)$, indefinite $(n=9)$, no sarcoidosis $(n=77)$. Of the 77 cases with no sarcoidosis, 44 were diagnosed with other diseases. The other 33 showed non-specific changes in the FNA and sarcoidosis was excluded by negative non-EUS pathology $(n=17)$ and clinical presentation. The sensitivity and specificity for EUS-FNA were $89 \%$ (95\% Cl 82 to 94) and $96 \%$ (95\% Cl 91 to 98), respectively, after exclusion of the indefinite cases in both groups.

Conclusions: EUS-FNA is an accurate method for diagnosing sarcoidosis in an unselected group of patients with mediastinal lymphadenopathy. The reported sensitivity and specificity must be appreciated in the context of the difficult and often incomplete clinical diagnosis of sarcoidosis.
S arcoidosis is a multisystem granulomatous disease of unknown aetiology. The prevalence of sarcoidosis ranges from less than one case to 40 cases per 100000 , with an age adjusted annual incidence rate in the US of 10.9 per 100000 for whites and 35.5 per 100000 for African Americans. ${ }^{1}$ Sarcoidosis can manifest in hilar and mediastinal lymph nodes, in the lungs, spleen, parotid and thyroid glands, central nervous system and in many other tissues. In asymptomatic stage I (bilateral hilar adenopathy on chest radiograph), a presumptive diagnosis can often be made based on a typical constellation of clinical and radiological findings, making histological diagnosis unnecessary. However, in symptomatic patients the diagnosis of sarcoidosis requires tissue confirmation of granulomatous inflammation and exclusion of infectious and malignant conditions. Although biopsy of easily accessible sites is often performed, more invasive procedures such as transbronchial biopsy (TBB) or mediastinoscopy may be necessary for diagnosis of mediastinal disease. Both procedures have shown a reasonable accuracy in diagnosing sarcoidosis ${ }^{2-5}$ but are associated with a significant rate of major complications. ${ }^{67}$ Furthermore, mediastinoscopy requires hospitalisation and general anaesthesia.

The value of cytological examination by fine needle aspiration (FNA) in the diagnosis of sarcoidosis has been shown in fine needle aspirates from various tissues affected by sarcoidosis. ${ }^{8-12}$ Several large series on endosonography guided FNA (EUS-FNA) of mediastinal lymph nodes mention sarcoidosis as a diagnosis in their specimens. ${ }^{13-19}$ Two papers have described in detail the value of EUS with FNA for establishing a diagnosis of sarcoidosis ${ }^{20}{ }^{21}$ and achieved a very high sensitivity and specificity. ${ }^{21}$ However, the number of patients in these studies was low and the pretest probability very high. In other words, EUS with FNA was used to confirm a nearly established clinical diagnosis.

In patients with mediastinal lymphadenopathy of unknown origin, sarcoidosis represents a possible differential diagnosis even though the clinical picture may not point to this conclusion. EUS with FNA has been described as a useful and safe diagnostic method for evaluating mediastinal lymphadenopathy of unknown origin. ${ }^{16} 1819$ As shown in these studies, EUS is able to image the entire mediastinum with the exception of the pretracheal region and portions of the paratracheal area. These studies achieved a high diagnostic yield in detecting malignant disease, whereas the ability to identify specific benign disease was dubious. There have been no data to show how accurately EUS-FNA identifies or excludes sarcoidosis in this setting.

\section{METHODS \\ Patients}

The EUS database of a single tertiary referral centre was reviewed for examinations with the indication and/or diagnosis of mediastinal lymphadenopathy of unknown origin from December 1995 to October 2002. With rare exceptions, the procedures were performed in an outpatient setting. Patients with suspected malignancy or a prior diagnosis of cancer were excluded, as were those with an established clinical diagnosis of sarcoidosis who underwent EUS with FNA solely for tissue confirmation.

Abbreviations: BAL, bronchoalveolar lavage; EUS-FNA, endosonography guided fine needle aspiration; TBB, transbronchial biopsy 
Clinical presentation at the time of the EUS, clinical follow up, and imaging studies were carefully reviewed by an expert in the field of sarcoidosis (MJ). In selected cases, clinical follow up had to be obtained from the patients themselves. The diagnosis of sarcoidosis was made based on a typical constellation of clinical findings and radiological imaging. The results of the EUS-FNA were not part of the decision making. Whenever available, other histological studies (TBB, mediastinoscopy, lung biopsy) were used to strengthen the diagnosis. Extensive efforts were made to confirm the diagnosis of either "sarcoidosis" or "no sarcoidosis". Cases which could not be attributed to either of the diagnoses were called "indefinite". After completion of this process, the diagnoses were compared with the results of the EUS-FNA.

The prospectively recorded data were analysed retrospectively. All patients gave written consent for EUS and EUSFNA. The Institutional Review Board (IRB) approved the protocol.

\section{Study design}

EUS was performed using either a curvilinear array (FG32UA, Pentax Precision Instruments, Orangeburg, NY, USA or GF-UC-140P, GF-UCT-140, Olympus America, Melville, NY, USA) or a mechanical sector scanner (GF-UM30P Olympus America). Initially, a mechanically rotating radial echoendoscope (GF-UM20, GF-UM130, GF-UM160, Olympus America) at frequencies of 7.5 and $12 \mathrm{MHz}$ was used prior to the curvilinear echoendoscope. This pattern was discontinued when more experience was gained using the curvilinear array to evaluate mediastinal anatomy. EUS-FNA was performed with the curvilinear array scanner and a 22 gauge, $14 \mathrm{~cm}$ adjustable length needle (Wilson-Cook Inc, Winston-Salem, NC, USA). Tru-cut biopsy was also performed with the curvilinear array scanner and a 19 gauge Tru-cut needle (Quick-Core; Wilson-Cook Inc). Each lymph node was sampled with a minimum of four needle passes unless a definitive diagnosis was given before the fourth pass.

Specimens were immediately prepared and preliminary results were given by a cytopathologist in the endoscopy suite. In cases where the samples demonstrated granulomatous inflammation but with non-diagnostic changes, special stains were performed as were cultures for acid-fast bacilli (AFB) and/or fungi. In cases where the preliminary on-site cytology showed a definitive non-granulomatous diagnosis, no stains or cultures were performed. The definitive cytological diagnosis of sarcoidosis was made by an experienced cytopathologist (MF) if non-caseating granulomas or other features consistent with granulomatous lymphadenitis were identified (figs 1 and 2).

\section{RESULTS}

One hundred and twenty seven patients ( 84 men) of mean age 55 years (range 19-85) met the inclusion criteria. In three patients clinical and radiological information was inadequate to make a diagnosis. The sensitivity and specificity of EUS-FNA in detecting sarcoidosis was therefore determined in 124 patients.

The demographic data of the study patients are shown in table 1 , which also summarises the clinical, radiological, and pathological features of the study population. It is noteworthy that there are no substantial differences between patients in whom granulomas were identified (group 1) and those with no granulomas (group 2). Patients with granulomas expressed slightly more constitutional symptoms and showed other clinical findings (uveitis, erythema nodosum, arthralgia) which may point to the diagnosis of sarcoidosis. Further diagnostic testing with angiotensin converting enzyme (ACE), calcium, and bronchoalveolar lavage (BAL) was carried out only in a small group of patients. Computed
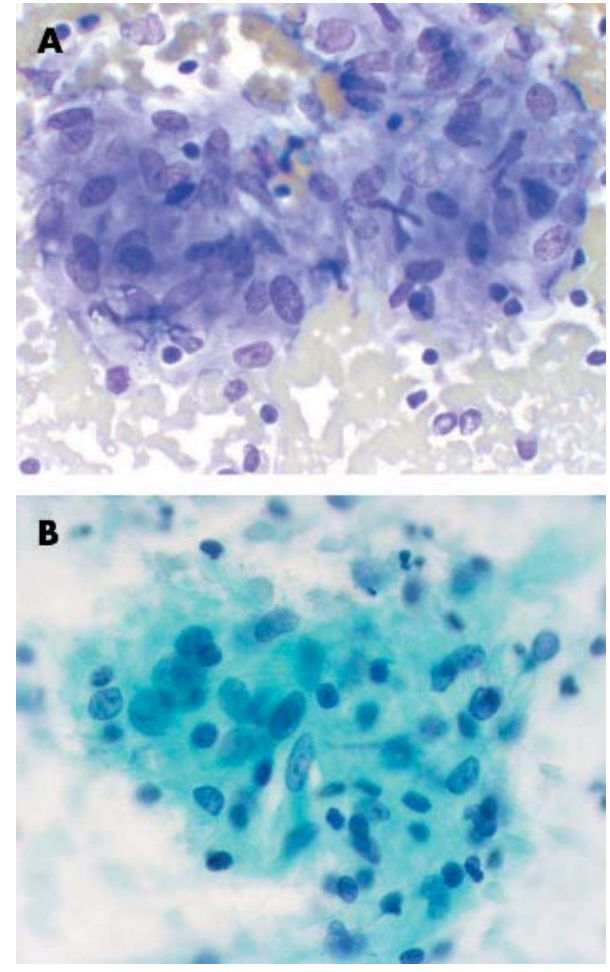

Figure 1 Cytological appearance of mediastinal lymph node obtained by EUS-FNA. Typical epitheloid granulomas in sarcoidosis with carrot shaped nuclei and abundant cytoplasm of epitheloid histiocytes in (A) modified Giemsa and (B) Papanicolaou stain.
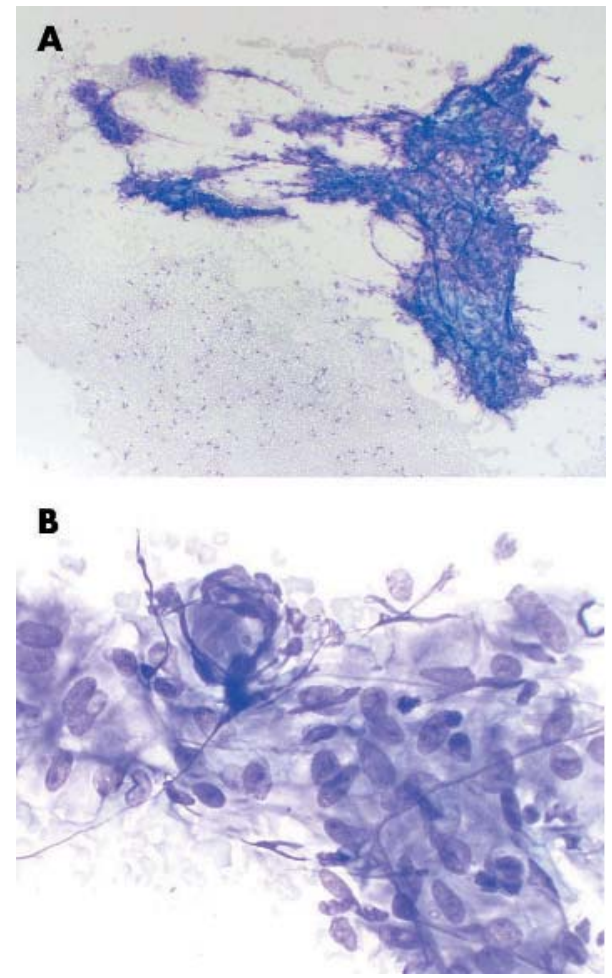

Figure 2 Cytological appearance of mediastinal lymph node obtained by EUS-FNA. (A) A tissue fragment of spindle cells that could be misinterpreted as fibrous tissue is in fact a poorly formed granuloma on higher magnification (B). 
Table 1 Demographic data and clinical characteristics of patients with mediastinal lymphadenopathy of unknown origin diagnosed by EUS

\begin{tabular}{|c|c|c|c|}
\hline & $\begin{array}{l}\text { Granulomas identified } \\
\text { by EUS-FNA } \\
(n=35)\end{array}$ & $\begin{array}{l}\text { No granulomas identified } \\
\text { by EUS-FNA } \\
(n=89)\end{array}$ & $\begin{array}{l}\text { Total with mediastinal } \\
\text { lymphadenopathy } \\
\text { ( } n=124)\end{array}$ \\
\hline \multicolumn{4}{|l|}{ Age (years) } \\
\hline Mean & 49 & 58 & 55 \\
\hline Range & $19-74$ & $24-85$ & $19-85$ \\
\hline \multicolumn{4}{|l|}{ Sex } \\
\hline Female & 17 & 25 & 42 \\
\hline Male & 18 & 64 & 82 \\
\hline \multicolumn{4}{|l|}{ Ethnic group } \\
\hline White & 19 & 64 & 83 \\
\hline Black & 15 & 25 & 40 \\
\hline Others & 1 & 0 & 1 \\
\hline \multicolumn{4}{|l|}{ Symptoms } \\
\hline Constitutional & 15 & 14 & $29(23 \%)$ \\
\hline Respiratory & 18 & 24 & $42(34 \%)$ \\
\hline Arthralgia & 2 & 3 & $5(4 \%)$ \\
\hline Erythema nodosum & 1 & 1 & $2(<2 \%)$ \\
\hline Organ dysfunction & 2 & - & $2(<2 \%)$ \\
\hline Other enlarged lymph nodes & - & 2 & $2(<2 \%)$ \\
\hline \multicolumn{4}{|l|}{ Radiology } \\
\hline \multicolumn{4}{|l|}{ Mediastinal lymph nodes } \\
\hline Positive & 32 & 80 & $112(90 \%)$ \\
\hline Negative & 3 & 9 & $12(10 \%)$ \\
\hline \multicolumn{4}{|l|}{ Hilar lymph nodes } \\
\hline Positive & 13 & 35 & $48(39 \%)$ \\
\hline Negative & 22 & 54 & $76(61 \%)$ \\
\hline \multicolumn{4}{|l|}{ Laboratory tests } \\
\hline \multicolumn{4}{|l|}{$\mathrm{ACE}$} \\
\hline Positive & 1 & 0 & 1 \\
\hline Negative & 2 & 4 & 6 \\
\hline \multicolumn{4}{|l|}{ Tissue sampling } \\
\hline \multicolumn{4}{|l|}{ TBB } \\
\hline Positive & 1 & - & 1 \\
\hline Negative & 5 & 18 & 23 \\
\hline \multicolumn{4}{|l|}{ Mediastinoscopy } \\
\hline Positive & 4 & 1 & 5 \\
\hline Negative & 3 & 4 & 7 \\
\hline \multicolumn{4}{|l|}{ Other biopsies } \\
\hline Positive & 2 & - & 2 \\
\hline Negative & 3 & 5 & 8 \\
\hline \multicolumn{4}{|l|}{ BAL } \\
\hline Positive & - & - & - \\
\hline Negative & 4 & 11 & 15 \\
\hline \multicolumn{4}{|l|}{ Treatment } \\
\hline Response to steroids & 6 & 2 & 8 \\
\hline
\end{tabular}

tomographic (CT) scanning of the chest identified most of the patients with mediastinal and hilar lymphadenopathy. In some patients the mediastinal lymphadenopathy was an incidental finding by EUS and therefore no prior diagnosis was made by other imaging techniques. In this small group of patients the EUS was performed for evaluation of other suspected benign disease (for example, gallstone disease, chronic pancreatitis). Nearly all patients had enlarged lymph nodes $(\geqslant 10 \mathrm{~mm})$ in the mediastinum (subcarina, AP window, paratracheal, para-aortal), whereas only one third had hilar lymphadenopathy. This proportion was the same in groups 1 and 2 .

After careful review of all the information, each case was assigned a diagnosis if possible. The diagnosis of sarcoidosis was made in 28 cases. In seven cases tissue confirmation was available. Interestingly, in three of these cases a negative TBB specimen was corrected by a positive mediastinoscopy. In the other 21 patients the diagnosis of sarcoidosis was made by a suggestive clinical and radiological follow up and/or a good response to steroid therapy. In 80 cases the diagnosis of sarcoidosis was excluded by another diagnosis $(n=47)$, by negative tissue diagnosis with negative follow up $(n=17)$, or negative follow up alone $(n=16)$. The mean follow up in patients with negative follow up alone was 18 months (range 2-62). In the remaining 16 cases some were suspicious for

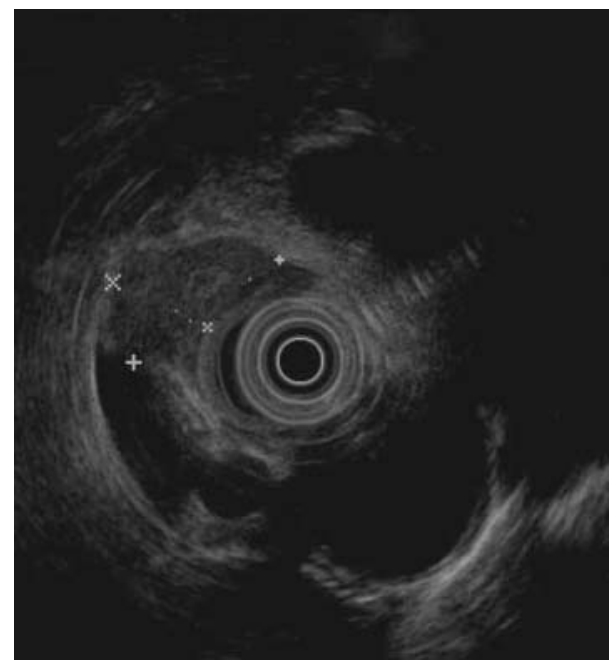

Figure 3 EUS image of mediastinal lymphadenopathy in the subcarina (in calipers), diagnosed as sarcoidosis. 


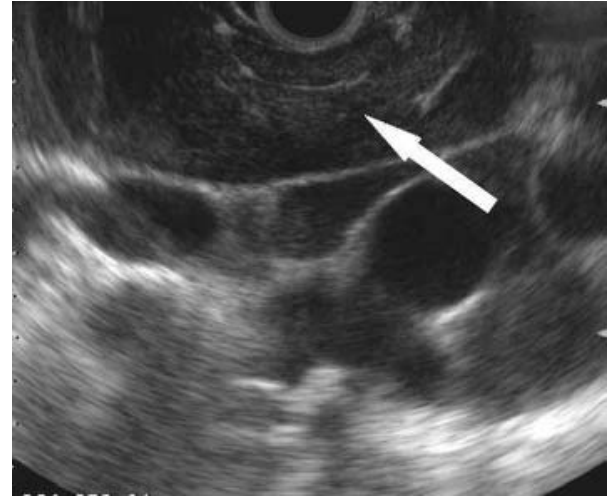

Figure 4 EUS image of mediastinal lymphadenopathy in the AP window (arrow), diagnosed as sarcoidosis.

sarcoidosis, others were unlikely to be sarcoidosis, but none could be definitively diagnosed. The mean follow up in these indefinite cases was 15 months (range 3-48).

As a consequence of the inclusion criteria, EUS detected enlarged mediastinal lymph nodes in all patients participating in the study. EUS guided FNA was performed in all patients. The cytological examination showed granulomas in 35 patients (group 1) and no granulomas in 89 patients (group 2). Most of the enlarged lymph nodes were found in the subcarina (84\%) and in the AP window (40\%) (figs 3 and 4). In a few patients, paratracheal and para-aortic lymph nodes were enlarged. In one third of patients more than one station in the mediastinum showed lymphadenopathy. The mean size of the enlarged lymph nodes was $29 \mathrm{~mm}$ in the long axis and $18 \mathrm{~mm}$ in the short axis. There was no substantial difference between the two groups in terms of the location and size of the lymph nodes (table 2). In 61 cases of EUS-FNA, additional needle passes were performed for AFB and fungal cultures. Thirty of the 35 patients (86\%) who had granulomatous findings at EUS-FNA had cultures obtained. In this group, cultures for $\operatorname{AFB}(\mathrm{n}=28)$ and/or fungus $(\mathrm{n}=28)$ were all negative (three were false negatives). In 31 of 55 patients $(56 \%)$ with non-diagnostic changes at EUSFNA, cultures for AFB and/or fungus were performed. In three cases tuberculosis $(n=2)$ or fungal disease $(n=1)$ were detected. In one case the diagnosis of tuberculosis was missed. In five patients an additional core biopsy of the mediastinal lymph node was performed. In all cases, FNA and core biopsy revealed the same result (granuloma $\mathrm{n}=2$, benign lymphocytes $n=3$ ). There were no complications associated with the procedures.
In the final phase of the study the definite diagnoses (sarcoidosis, no sarcoidosis, indefinite) were compared with the results of the EUS-FNA, including the results of cytological examinations and cultures (fig 5). Of the 35 patients identified with granulomas by EUS-FNA, 25 had confirmed sarcoidosis, seven were indefinite ("some suspicion for sarcoidosis"), and three had false positive diagnoses of sarcoidosis. In all three cases the underlying infectious disease was not detected by cultivation of the aspirate. Of the 89 patients without granulomas by EUS-FNA, three had sarcoidosis, nine were indefinite ("sarcoidosis unlikely"), and in 77 cases sarcoidosis was excluded. All three patients with missed sarcoidosis had a very typical clinical follow up (constitutional and respiratory symptoms, erythema nodosum, arthralgia). Two of them responded promptly to steroid therapy and one had positive tissue confirmation by mediastinoscopy. The exclusion of sarcoidosis $(n=77)$ was established by another diagnosis, negative follow up and/or negative tissue diagnosis. EUS-FNA misdiagnosed 10 out of 44 other diagnoses. In four out of six patients the diagnosis of lymphoma was missed. In two of them flow cytometry and immunocytochemistry of the aspirate was not performed.

The sensitivity and specificity for EUS-FNA in detecting sarcoidosis was $89 \%$ (95\% CI 82 to 94 ) and 96\% (95\% CI 91 to 98), respectively, after exclusion of the indefinite cases in both groups. Assuming the worst case-that is, all indefinite cases are false negative and false positive-the sensitivity and specificity would be $68 \%$ (95\% CI 59 to 77 ) and $89 \%$ (95\% CI 82 to 94$)$, respectively.

\section{DISCUSSION}

The results of this study show that EUS with FNA is an accurate method for diagnosing sarcoidosis in an unselected group of patients with mediastinal lymphadenopathy of unknown origin. The sensitivity and specificity were $89 \%$ and $96 \%$ after exclusion of the indefinite cases in both groups. These values must be appreciated in the context of the difficult and often incomplete clinical diagnosis of sarcoidosis.

Mediastinal lymphadenopathy represents a diagnostic challenge because of the variety of potential pathological aetiologies and the difficulty of access for tissue sampling. Different diagnostic modalities have been used for evaluating mediastinal lymphadenopathy of undetermined origin including CT, MRI, bronchoscopy with TBB, mediastinoscopy, and thoracotomy. Evaluation of the mediastinum using EUS-FNA has been found to be useful for a number of indications. Several publications have shown that EUS-FNA establishes the diagnosis in a high percentage of patients with mediastinal lymphadenopathy of unknown origin. ${ }^{16} 1819$

Table 2 EUS characteristics of mediastinal lymphadenopathy of unknown origin and results of AFB and fungal cultures

\begin{tabular}{|c|c|c|c|}
\hline & $\begin{array}{l}\text { Granuloma identified } \\
\text { by EUS-FNA }(n=35)\end{array}$ & $\begin{array}{l}\text { No granuloma identified } \\
\text { by EUS-FNA ( } n=89 \text { ) }\end{array}$ & $\begin{array}{l}\text { Total with mediastinal } \\
\text { lymphadenopathy }(n=124)\end{array}$ \\
\hline \multicolumn{4}{|l|}{ LN station } \\
\hline Subcarina & 34 & 70 & 104 (84\%) \\
\hline AP window & 14 & 36 & $50(40 \%)$ \\
\hline Paratracheal & 2 & 9 & $11(9 \%)$ \\
\hline Para-aortal & 6 & 15 & 21 (17\%) \\
\hline \multicolumn{4}{|l|}{ Size of LN (mm) } \\
\hline Long axis & $33(15-60)$ & $28(8-68)$ & $29(8-68)$ \\
\hline Short axis & $19(6-40)$ & $17(5-40)$ & $18(5-40)$ \\
\hline \multicolumn{4}{|l|}{ AFB culture } \\
\hline Positive & - & 2 & 2 \\
\hline Negative & 28 & 29 & 57 \\
\hline \multicolumn{4}{|l|}{ Fungal culture } \\
\hline Positive & - & 1 & 1 \\
\hline Negative & 28 & 23 & 51 \\
\hline
\end{tabular}




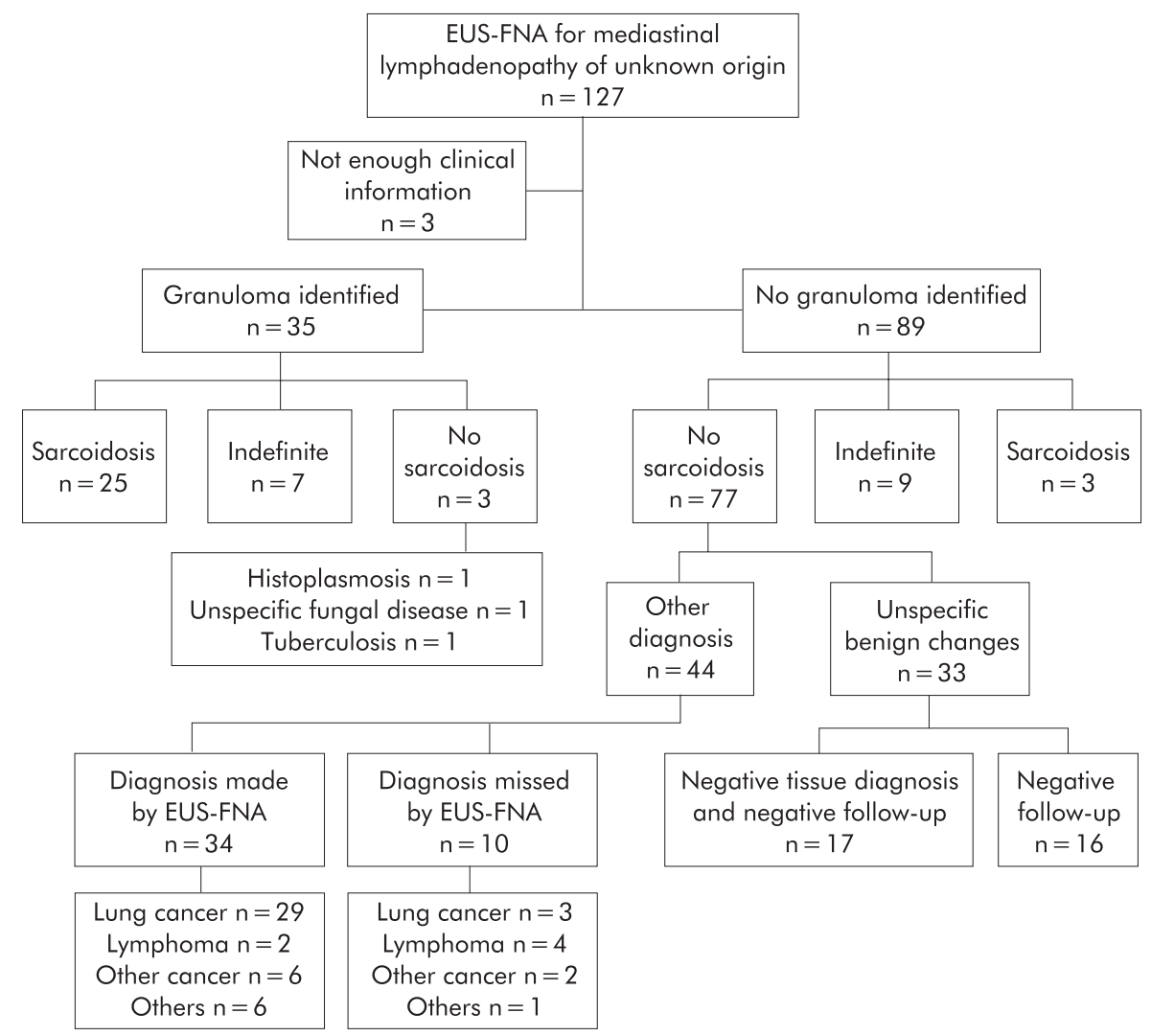

Figure 5 Patients with mediastinal lymphadenopathy of unknown origin undergoing EUS with FNA.

Most notably, very promising results have been reported for EUS-FNA in detecting posterior mediastinal lymph node metastasis in patients with non-small cell lung cancer. ${ }^{22-26}$ EUS-FNA can also provide cytological specimens diagnostic for lymphoma, as long as flow cytometry and immunocytochemistry is performed. ${ }^{17}$

In most of these studies sarcoidosis represented a small subset of the diagnoses. To date, only two studies have dealt specifically with the diagnosis of sarcoidosis by EUS and EUS-FNA. ${ }^{20}{ }^{21}$ Mishra et al investigated seven patients with mediastinal lymphadenopathy in whom EUS and EUS-FNA helped to confirm the diagnosis of sarcoidosis. ${ }^{20}$ The nodal characteristics on EUS were very similar in all seven patients-elongated, triangular, and draped around the oesophagus. They appeared to be irregularly shaped and hypoechoic with linear hyperechoic foci. The mean number of lymph nodes was 2.8 per patient, and most of the patients had enlarged lymph nodes in the subcarina and the AP window. The mean size of the long and short axes was $3.4 \mathrm{~cm}$ and $2.5 \mathrm{~cm}$, respectively. The authors concluded that large irregular shaped nodes are suggestive for sarcoidosis. Fritscher-Ravens et al achieved a sensitivity of $100 \%$ and specificity of $94 \%$ for EUS-FNA in the diagnosis of sarcoidosis in 19 patients with a very high pretest probability. ${ }^{21}$ In one patient the final diagnosis was tuberculosis. This diagnosis was also made by EUS-FNA and mycobacterial cultures, respectively. As reported by the authors, the typical EUS morphology observed in sarcoidosis represents either isoechoic or hypoechoic lymph nodes and atypical vascular structures within them in a subset of patients.

However, the number of patients in these two studies was low and the pretest probability very high. All patients had typical findings on chest radiographs or chest CT scans. Most of the patients had either typical symptoms or extrapulmonary complaints (erythema nodosum, parotiditis, retinal vasculitis), or other findings that are consistent with sarcoidosis (hypercalcaemia, raised ACE, raised CD4/CD8 ratio in BAL fluid, positive TBB). EUS with FNA was therefore used to confirm a nearly established clinical diagnosis. All patients included in these studies showed granulomatous inflammation in the cytological specmens obtained by EUS-FNA. It seems that the positive diagnosis of granuloma was chosen as an inclusion criterion. As a consequence of this design, the calculation of the sensitivity cannot be performed properly. These two studies were clearly designed to illustrate the value of EUS-FNA in confirming an already established diagnosis of sarcoidosis. They do not answer the question "How accurately does EUS-FNA include or exclude the diagnosis of sarcoidosis in patients with mediastinal lymphadenopathy of unknown origin?".

This retrospective study also has limitations. Firstly, the study lacks an accurate gold standard. The clinical diagnosis of sarcoidosis can be very difficult and is often incomplete. After exclusion of malignant disease in an asymptomatic patient, the tissue diagnosis is often not aggressively pursued and clinical follow up is thought to be sufficient. Using this strategy, asymptomatic sarcoidosis remains underdiagnosed. An ideal study would be prospective so that all patients would undergo tissue confirmation after EUS-FNA. As a consequence, all participants would have to undergo invasive tissue diagnosis (by mediastinoscopy, CT guided biopsy, etc), even after exclusion of malignancy by EUS-FNA. Such a study is not ethically justifiable. A retrospective design therefore had to be used. To overcome the limitations of a retrospective study, every effort was made to collect as much clinical information as possible. Nevertheless, in a significant number of cases there was no definitive diagnosis due to missing or conflicting clinical data. In some of these cases no further tissue diagnostic tests were performed because the referring physicians relied upon the diagnosis made by 
EUS-FNA. Cases with an indefinite diagnosis were excluded from the calculation of the sensitivity and specificity. This may be subject to bias; in particular, the sensitivity may be overestimated. In a worst case scenario where all indefinite cases are considered false negative and false positive, the sensitivity and specificity would be $68 \%$ and $89 \%$, respectively.

In conclusion, EUS-FNA is an accurate method for diagnosing sarcoidosis in an unselected group of patients with mediastinal lymphadenopathy. The reported sensitivity and specificity must be appreciated in the context of the difficult and often incomplete clinical diagnosis of sarcoidosis. From previous studies we have learned that EUS-FNA is a very accurate tool for diagnosing or excluding malignancy in mediastinal lymphadenopathy. The findings of this study may be helpful for interpreting non-malignant cytological specimens obtained from enlarged mediastinal lymph nodes.

\section{Authors' affiliations}

S M Wildi, W E Fickling, N Schmulewitz, S Varadarajulu, S S Roberts, P Prasad, R H Hawes, M B Wallace, B J Hoffman, Digestive Disease Center, Medical University of South Carolina, Charleston, SC 29425, USA

M A Judson, Department of Pulmonary and Critical Care Medicine, Medical University of South Carolina, Charleston, SC 29425, USA

M Fraig, Department of Pathology, Medical University of South Carolina, Charleston, SC 29425, USA

SMW was supported by a grant from the Swiss National Science Foundation.

Presented as a poster at the Digestive Disease Week 2003 in Orlando.

\section{REFERENCES}

1 Rybicki BA, Major M, Popovic J Jr, et al. Racial differences in sarcoidosis incidence: a 5-year study in a health maintenance organization. Am J Epidemiol 1997; 145:234-41.

2 Koerner SK, Sakowitz AJ, Appelman RI, et al. Transbronchial lung biopsy for the diagnosis of sarcoidosis. N Engl J Med 1975;93:268-70.

3 Koontz CH, Joynes LR, Nelson RA. Transbronchial lung biopsy via the fiberoptic bronchoscope in sarcoidosis. Ann Intern Med 1976;85:64-6.

4 Mitchell DM, Mitchell DN, Collins JV, et al. Transbronchial lung biopsy through fiberoptic bronchoscope in diagnosis of sarcoidosis. BMJ 1980;280:429-32.

5 Mikhail JR, Shepherd M, Mitchell DN. Mediastinal lymph node biopsy in sarcoidosis. Endoscopy 1979;1:5-8

6 Harrow EM, Oldenburg FA, Lingenfelter MS, et al. Transbronchial needle aspiration in clinical practice: a five-year experience. Chest 1989;96:1268-72.
7 Reich JM, Brouns MC, Connor EA, et al. Mediastinoscopy in patients with presumptive stage I sarcoidosis: a risk/benefit, cost/benefit analysis. Chest 1998; 113:147-53.

8 Pisircriler R, Atay Z, Lang W. Cytological diagnosis of intrathoracic epitheloid cellular inflammatory process. Pneumologie 1990;44:767-70.

9 Pauli G, Pelletier A, Bohner C, et al. Transbronchial needle aspiration in the diagnosis of sarcoidosis. Chest 1984;85:482-4.

10 Selroos O, Koivunen E. Usefulness of fine-needle aspiration biopsy of spleen in diagnosis of sarcoidosis. Chest 1983;83:193-5.

11 Lohela P, Tikkakoski T, Strengell L, et al. Ultrasound-guided fine-needle aspiration cytology of non-palpable supraclavicular lymph nodes in sarcoidosis. Acta Radiol 1996;37:896-9.

12 Tambouret R, Geisinger KR, Powers CN, et al. The clinical application and cost analysis of fine-needle aspiration biopsy in the diagnosis of mass lesions in sarcoidosis. Chest 2000;117:1004-11.

13 Wiersma MJ, Vilmann P, Giovannini M, et al. Endosonography-guided fineneedle aspiration biopsy: diagnostic accuracy and complication assessment. Gastroenterology 1997; 1 12:1087-95.

14 Giovannini M, Seitz JF, Monges G, et al. Fine-needle aspiration cytology guided by endoscopic ultrasonography: results in 141 patients. Endoscopy 1995;27:171-7.

15 Huehnerbein M, Dohmoto M, Haensch W, et al. Endosonography-guided biopsy of mediastinal and pancreatic tumors. Endoscopy 1998;30:32-6.

16 Fritscher-Ravens A, Sriram PVJ, Bobrowski C, et al. Mediastinal lymphadenopathy in patients with or without previous malignancy: EUS-FNAbased differential cytodiagnosis in 153 patients. Am J Gastroenterol 2000;95:2278-84.

17 Ribeiro A, Vazquez-Sequeiros E, Wiersma LM, et al. EUS-guided fine-needle aspiration combined with flow cytometry and immunochemistry in the diagnosis of lymphoma. Gastrointest Endosc 2001;53:485-91.

18 Devereaux BM, LeBlanc JK, Yousif E, et al. Clinical utility of EUS-guided fineneedle aspiration of mediastinal masses in the absence of known pulmonary malignancy. Gastrointest Endosc 2002;56:397-401.

19 Catalano MF, Nayar R, Gress F, et al. EUS-guided fine needle aspiration in mediastinal lymphadenopathy of unknown etiology. Gastrointest Endosc 2002;55:863-9.

20 Mishra G, Sahai AV, Penman ID, et al. Endoscopic ultrasonography with fineneedle aspiration: an accurate and simple diagnostic modality for sarcoidosis. Endoscopy 1999;31:377-82

21 Fritscher-Ravens A, Sriram PVJ, Topalidis T, et al. Diagnosing sarcoidosis using endosonography-guided fine-needle aspiration. Chest 2000; 1 18:928-35.

22 Panelli F, Erickson RA, Prasad VM. Evaluation of mediastinal masses by endoscopic ultrasound and endoscopic ultrasound-guided fine needle aspiration. Am J Gastroenterol 2001;96:401-8.

23 Gress FG, Savides TJ, Sandler A, et al. Endoscopic ultrasonography, fineneedle aspiration biopsy guided by endoscopic ultrasonography, and computed tomography in the preoperative staging of non-small-cell lung cancer: a comparative study. Ann Intern Med 1997; 127:604-12.

24 Silvestri GA, Hoffman BJ, Bhutani MS, et al. Endoscopic ultrasound with fineneedle aspiration in the diagnosis and staging of lung cancer. Ann Thorac Surg 1996:61:1441-5.

25 Wallace MB, Silvestri GA, Sahai AV, et al. Endoscopic ultrasound-guided fine needle aspiration for staging patients with carcinoma of the lung. Ann Thorac Surg 2001;72:1861-7.

26 Fritscher-Ravens A, Bohuslavizki KH, Brandt L, et al. Mediastinal lymph node involvement in potentially resectable lung cancer: comparison of $\mathrm{CT}$, positron emission tomography, and endoscopic ultrasonography with and without fineneedle aspiration. Chest 2003;123:442-51. 